differences in body weight. However, no effects of body weight on the immune responses were seen. The effect of sire was highly significant $(p \leq 0.001)$ indicating a genetic influence on the immune response. The overall correlation between the primary and secundary response was for K88 antigen 0.76 and for 0149 antigen 0.15 .

\title{
Heterogeneity of RNA transcription activities of immune cells of domestic animals
}

\author{
B. MAYR and W. SCHLEGER \\ Institut für Tierzucht und Genetik, Veterinärmedizinische Universität Wien \\ Linke Bahngasse 11, 1030 Wien, Austria
}

Nucleolus organizer regions were determined in cattle, pig, sheep, goat, dog, horse and chicken. The mapped genes were correlated to the nucleoli formed in peripheral blood lymphocyte interphases. A positive correlation was found between the number of nucleolar organizer regions per diploid genome and the nucleolar coefficient. Peculiarities of the different species concerning nucleolar formation and association/dissociation pattern is highlighted. The use in definition of immune cells in domestic animals in order to investigate cellular heterogeneity is pointed out.

\section{Immunoglobulin levels in the blood serum of pigs as criteria of heredity and ontogenesis}

\author{
E. WEHRHAHN, F. KLOBASA and F. HABE \\ Institut für Tierzucht $u$. Tierverhalten, Mariensee \\ 3057 Neustadt 1, West Germany
}

Milk and blood immunoglobulins were analyzed in 22 young sows and in 24 older sows (DL). In addition, the blood immunoglobulin levels of two offsprings each were monitored. Older sows had higher total Ig-serum values than young sows. Their milk IgG and IgA contents also surpassed those of young sows, which however showed higher IgM contents. The initial serum pattern of the piglets mirrored the maternal Ig-secretions in the milk (passive immunization). After weeks 2-3 piglets of the young sows showed a steeper rise in the Ig production rate than piglets from older sows. They caught-on however during the fattening period and had arrived at higher IgA-levels when slaughtered.

A number of correlations between sow/piglet Ig patterns, fattening performance and carcass compositions will be reported.

\section{Hypothermia in newborn lambs}

\author{
A. EALES \\ Moredun Research Institute, 408 Gilmerton Road, Edinburgh EH17 $7 J H$
}

Hypothermia, a low body temperature, is an important cause of mortality in newborn lambs in the U.K. There are two major causes of this condition : (1) Excessive heat loss 
from lambs aged up to four hours and (2) Depressed heat production due to starvation in older lambs. All lambs are susceptible to hypothermia but high risk lambs include twins and triplets, lambs which suffer hypoxia during birth, lambs from ewes in poor condition and lambs from very young or very old ewes. Prevention of hypothermia depends on good nutrition during pregnancy and a high standard of management at lambing time. Effective treatment of hypothermia depends on early detection of the condition, administration of glucose to lambs aged five hours of more, rewarming in warm air and careful aftercare.

\title{
The effect of ACTH on parturition as defence mechanism stimulator in sows
}

\author{
J.B. LUDVIGSEN \\ National Institute of Animal Science \\ Rolighedsvej 25, 1958 Copenhagen V, Denmark
}

Injection of a long acting ACTH preparation on the 140th day of pregnancy reduces parturition time by 21 and 23 p. 100 in Danish Landrace and Piétrain sows respectively. The parturition time of the Piétrain sows was about 20 p. 100 longer than in the Danish Landrace.

The difference in parturition time between the two breeds, and the shortening effect of ACTH is discussed from general stress susceptibility points of view : the Piétrain having a higher incidence of PSE, PSS and Halothane positivity than Danish Landrace, caused by an impaired genetic ability to mobilize adaptive endocrine systems, especially the pituitaryadrenal cortical axis at time of parturition.

ACTH stimulates the adaptive system by way of an increased release of adrenal cortical steroids. One of the effects of an increase in circulating adrenal cortical hormones, is a stimulation of uterine contractions.

The mode of action of adrenal cortical hormones in the defence mechanism of the organism against external as well as internal stress situations, in the present case parturition, is discussed.

\section{Effect of $\mathbf{T}_{2}$ Fusarium toxin on adrenocortical function and immune response of growing pigs}

\author{
P. RAFAI and S. TUBOLY \\ University of Veterinary Science \\ H - 1400 Budapest, P.O. Box 2, Hungary
}

Six Hungarian Large White $\times$ Duroc $\mathrm{F}_{1}$ pigs fed $\mathrm{T}_{2}$ toxin at $5 \mathrm{mg} / \mathrm{kg}$ dietary level for 25 days after weaning at 28 days of age were examined for weight gain, adrenocortical activity and main cell-mediated and humoral immune reactions by comparison to 6 control pigs of the same age and breed.

Toxin treatment accounted for $49-77$ p. 100 feed refusal, and consequent marked depression of weight gain. The average daily toxin ingestion was assessed as $1.27 \mathrm{mg}$.

$\mathrm{T}_{2}$ also depressed the total leucocyte count, but did not give rise to leucocytopenia during the 25 days of treatment.

The adrenocortical activity of $T_{2}$-treated pigs increased significantly over the control. 